\title{
Cavity Closure of 2-Hydroxypropyl- $\beta$-cyclodextrin: Replica Exchange Molecular Dynamics Simulations
}

\author{
Khanittha Kerdpol ${ }^{1}$, Jintawee Kicuntod ${ }^{2}$, Peter Wolschann ${ }^{2,3,4}$, Seiji Mori ${ }^{5}$, Chompoonut \\ Rungnim ${ }^{6}$, Manaschai Kunaseth ${ }^{6}$, Hisashi Okumura ${ }^{7}$, Nawee Kungwan ${ }^{1,8, *}$ and Thanyada \\ Rungrotmongkol 2,9,10,*
}

1 Department of Chemistry, Faculty of Science, Chiang Mai University, Chiang Mai 50200, Thailand; khanittha.view@gmail.com and naweekung@gmail.com

2 Structural and Computational Biology Research Unit, Department of Biochemistry, Faculty of Science, Chulalongkorn University, Bangkok 10330, Thailand; jintawee.ki@gmail.com and thanyada.r@chula.ac.th

3 Department of Pharmaceutical Chemistry, University of Vienna, Vienna 1090, Austria; karl.peter.wolschann@univie.ac.at

4 Institute of Theoretical Chemistry, University of Vienna, Vienna 1090, Austria; karl.peter.wolschann@univie.ac.at

5 Institute of Quantum Beam Science, Graduate School of Science and Engineering, Ibaraki University, 2-1-1 Bunkyo, Mito, Ibaraki 310-8512, Japan; seiji.mori.compchem@vc.ibaraki.ac.jp

6 National Nanotechnology Center (NANOTEC), National Science and Technology Development Agency (NSTDA), Pathum Thani 12120, Thailand; chompoonut@nanotec.or.th and manaschai@nanotec.or.th

7 Research Center for Computational Science, Institute for Molecular Science, Okazaki, Aichi 444-8585, Japan; hokumura@ims.ac.jp

8 Center of Excellence in Materials Science and Technology, Chiang Mai University, Chiang Mai 50200, Thailand; naweekung@gmail.com

9 Ph.D. Program in Bioinformatics and Computational Biology, Faculty of Science, Chulalongkorn University, Bangkok 10330, Thailand; thanyada.r@chula.ac.th

${ }_{10}$ Molecular Sensory Science Center, Faculty of Science, Chulalongkorn University, 254 Phayathai Road, Patumwan, Bangkok 10330, Thailand; thanyada.r@chula.ac.th

* Correspondence: naweekung@gmail.com; Tel.: +66-5394-3341 ext. 101. (+66-5389-2277) and thanyada.r@chula.ac.th; Tel.: +66-2218-5426 (+66-2218-5418)

\begin{abstract}
Hydroxypropyl- $\beta$-cyclodextrin (HP $\beta C D$ ) has unique properties to enhance the stability and the solubility of low water-soluble compounds by inclusion complexation. Understanding of the structural properties of $\mathrm{HP} \beta C D$ and its derivatives based on the number of 2-hydroxypropyl (HP) substituents at the a-D-glucopyranose subunits is rather important. In this work, replica exchange molecular dynamics simulations were performed to investigate the conformational changes of single- and double-sided HP-substitution called as 6-HP $\beta$ CDs and 2,6-HP $\beta C D$, respectively. The results show that glucose subunits in both 6-HP $\beta$ CDs and 2,6-HP $\beta$ CDs have lower chance to flip than in $\beta C D$. Also, HP groups are occasionally blocking the hydrophobic cavity of HP $\beta C D$ s, thus hindering the drug inclusion. We found that HP $\beta C D$ s with high number of HPsubstitutions are more likely to be blocked, while HP $\beta C D$ s with double-sided HP-substitution are even more probable to be blocked. Overall, 6-HP $\beta C D$ s with three and four HP-substitutions are highlighted as the most suitable structures for guest encapsulation based on our conformational analyses such as structural distortion, radius of gyration, circularity and cavity self-closure of the HP $\beta$ CDs.
\end{abstract}

Keywords: 2-Hydroxypropyl- $\beta$-cyclodextrin (HP $\beta C D)$; Replica exchange molecular dynamics (REMD); Conformational change; Cavity self-closure

\section{Introduction}


Cyclodextrins (CDs) are cyclic oligosaccharides, which received much attention in various technical applications due to their unique properties. In the pharmaceutical industry, they are widely used to improve the stability and the solubility of insoluble drugs in water or organic solvent-water mixtures by molecular encapsulation [1-9]. The ability of encapsulation of CDs with drugs strongly depends on the structural nature of the applied CDs. There are several different kinds of CDs defined by number of glucose units and the most common CDs are $\alpha-, \beta-$, and $\gamma-C D$, with different number of a-D-glucopyranose units $(\alpha=6, \beta=7$ and $\gamma=8)$. Among these CDs, a derivative of $\beta$-cyclodextrin $(\beta C D)$ named as 2-hydroxypropyl- $\beta$-cyclodextrin (HP $\beta C D)$, shown in Figure 1, has been found to be more soluble and has a lower toxicity than $\beta C D$ [10-13].

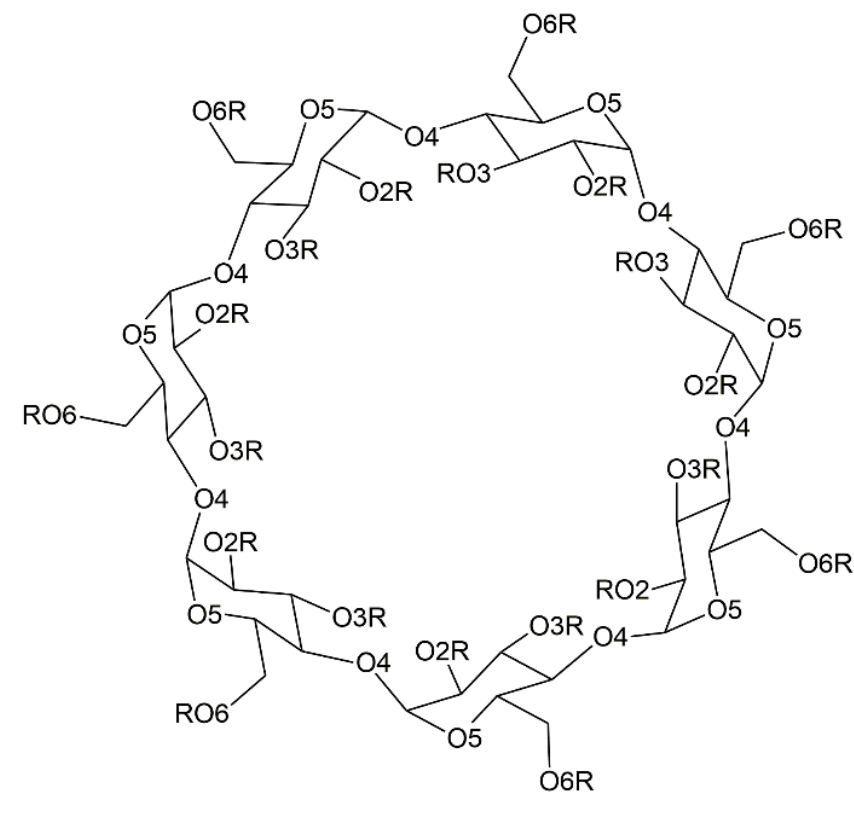

$\mathrm{R}=$ Hydrogen $(-\mathrm{H})$ or 2-Hydroxypropyl $\left(\mathrm{HP},-\mathrm{CH}_{2} \mathrm{CH}(\mathrm{OH}) \mathrm{CH}_{3}\right)$

2-Hydroxypropyl- $\beta$-cyclodextrin

Figure 1. Schematic representation of 2-hydroxypropyl- $\beta$-cyclodextrin $(\mathrm{HP} \beta C D)$, which comprises seven glucopyranose units.

$\mathrm{HP} \beta C D$, a partially substituted poly(hydroxypropyl) ether of $\beta C D$, is commercially available as a mixture with a certain range of degree of substitution [11,13-16]. The separation of individual derivatives and isomers from each other is rather difficult, in particular at industrial scale. Also, a selective synthesis of the individual isomer is not easy; therefore, a characterization of various derivatives with regard to their inclusion ability is not possible. However, theoretical studies particularly molecular dynamics simulations have become a popular tool to provide this important information. By investigating the molecular structural behavior at atomistic level of each substituted derivative, compounds with a certain degree of substitution of HP $\beta C D$ can be evaluated. Note that in the technical product, the degree of substitution exists randomly with different numbers of HP at other positions (e.g. at different glucose units at 2,3, and 6 position), which depends on the concentration of alkaline solution during the synthesis. Normally, $\beta C D$ alkylations are observed at the $\mathrm{O} 2$ and $\mathrm{O} 6$ positions $[13,17,18]$. Therefore, $\mathrm{HP} \beta \mathrm{CD}$ with substitutions at $\mathrm{O} 2$ (the most acidic position) and $\mathrm{O} 6$ (the most accessible position) with different degrees of substitution have been intensively investigated [11,13-16,19-22].

Generally, it is difficult to explore the conformations of biomolecules having complicated free energy surfaces with a larger number of local minima by single conventional simulation at low temperature condition. To overcome this problem, the replica-exchange molecular dynamics simulations (REMD) [23,24], which is one of the most effective method with generalized-ensemble algorithms, is applied by non-interacting replicas exchange at various temperatures [23-27]. This could allow us to obtain the information of the temperature dependence. In the present study, we applied the REMD method on $\beta C D$ and HP $\beta C D$ s models to study the conformational change affected by the different numbers of HP-substitutions on $\mathrm{O} 2$ and/or $\mathrm{O} 6$ atoms. The structural behaviors of all 
models were analyzed based on structural distortion analysis, radius of gyration, circularity and cavity self-closure of the HP $\beta C D$ s. By doing so, the best candidate of HP $\beta C D$ s with suitable amount of HP-substitution, representing the perfect conical shape for molecular encapsulation, will be provided as an useful guideline of suitable substitution degree of $\mathrm{HP} \beta C D$.

\section{Computational Methods}

The optimized structures of native $\beta C D$ and Hep6-HP $\beta C D$ were taken from our previous studies $[28,29]$. The other HP $\beta C D$ derivatives were prepared by the different numbers of 2-hydroxypropyl (HP) substitutions at the $\mathrm{O} 2$ or $\mathrm{O} 6$ positions on a-D-glucopyranose units with substitution degree around 0.14-1.14 from one to eight HP-substitutions on $\beta C D$ as shown in Figure 2. The HPsubstitutions on $\beta C D$ in this work were divided into two groups, single- and double-sided substitutions. For single-sided HP-substitution (Figure 2a), the HP groups were substituted one up to seven HP only on $\mathrm{O} 6$ atoms of the primary rim (called as 6-HPßCDs) because the O6 is more reactive compared to $\mathrm{O} 2$ atoms of the secondary rim. In the case of double-sided HP-substitution (Figure 2b), the structures were generated by introducing one up to four HP groups at the $\mathrm{O} 2$ atoms of the non-substituted glucose units (called as 2,6-HP $\beta C D$ ), as defined in Table 1.

(a) Single-sided HP-substitution

(b) Double-sided HP-substitution
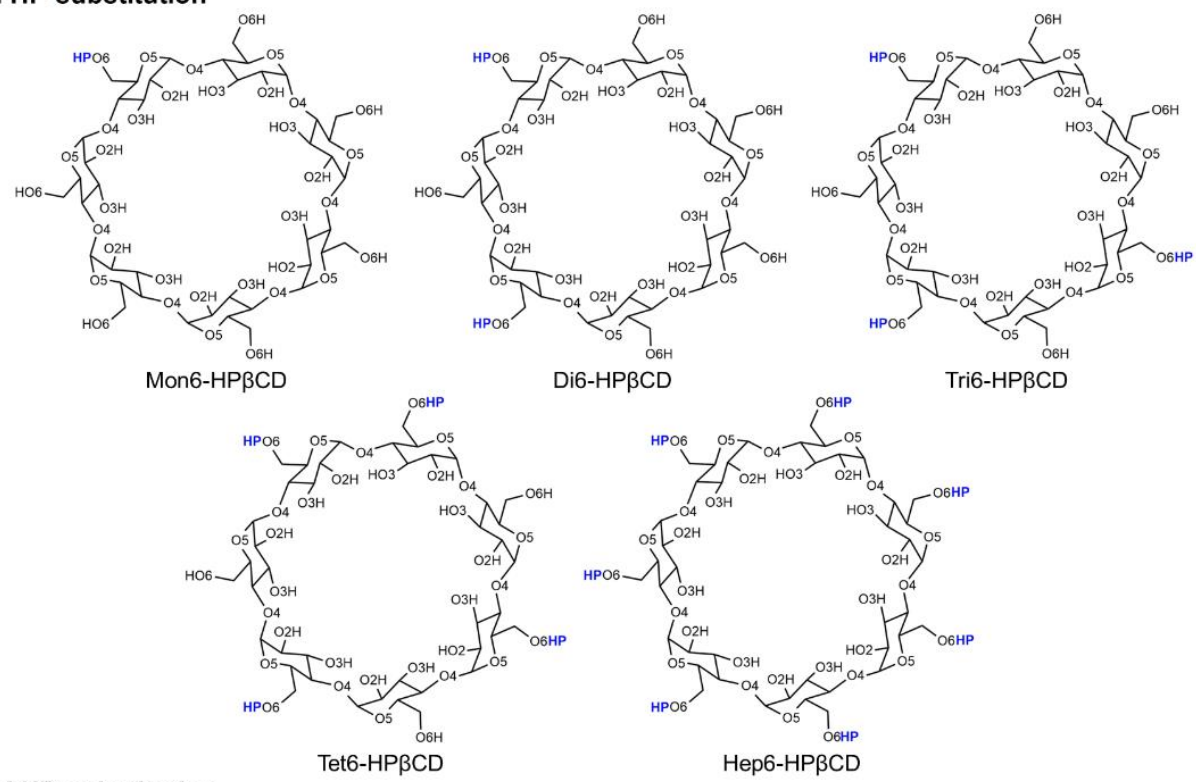

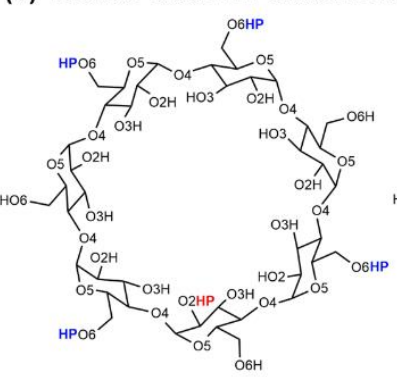

Mon2Tet6-HPBCD

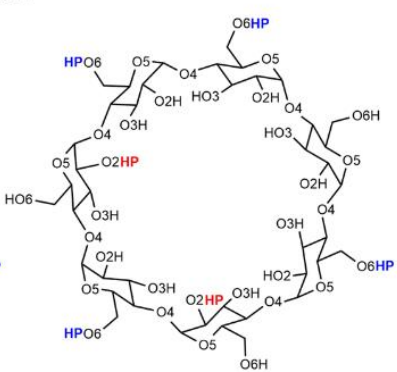

Di2Tet6-HPBCD

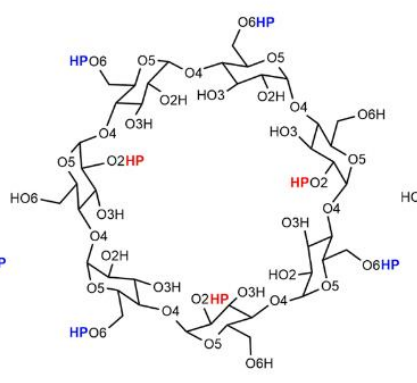

Tri2Tet6-HP $\beta C D$

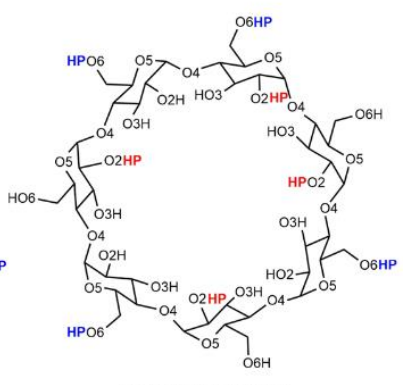

Tet2Tet6-HPßCD

Figure 2. Models introducing HP groups at O2 (red) and/or O6 (blue) positions on glucose subunits.

Table 1. Model summary of introducing HP groups at the $\mathrm{O} 2$ and/or O6 positions on glucose subunits.

\begin{tabular}{lcccc}
\hline \multicolumn{2}{c}{ Models } & Molar substitution & O2 substitution & O6 substitution \\
\hline$\beta C D$ & 0.00 & None & None \\
Single-sided HP-substitution & & & \\
Mon6-HP $\beta C D$ & 0.14 & None & 1 (At glucose unit 1)
\end{tabular}




\begin{tabular}{|c|c|c|c|}
\hline Di6-HP $\beta C D$ & 0.28 & None & 2 (At glucose units 1 and 3 ) \\
\hline Tri6-HP $\beta C D$ & 0.43 & None & 3 (At glucose units 1, 3 and 5) \\
\hline Tet6-HP $\beta C D$ & 0.57 & None & 4 (At glucose units $1,3,5$ and 7$)$ \\
\hline Hep6-HP $\beta C D$ & 1.00 & None & 7 (At all glucose units) \\
\hline \multicolumn{4}{|c|}{ Double-sided HP-substitution } \\
\hline Mon2Tet6-HP $\beta C D$ & 0.71 & 1 (At glucose unit 4$)$ & 4 (At glucose units $1,3,5$ and 7$)$ \\
\hline Di2Tet6-HP $\beta C D$ & 0.85 & 2 (At glucose units 2 and 6 ) & 4 (At glucose units $1,3,5$ and 7$)$ \\
\hline Tri2Tet6-HP $\beta C D$ & 1.00 & 3 (At glucose units 2, 4 and 6) & 4 (At glucose units $1,3,5$ and 7$)$ \\
\hline Tet2Tet6-HP $\beta C D$ & 1.14 & 4 (At glucose units $2,4,6$ and 7 ) & 4 (At glucose units $1,3,5$ and 7$)$ \\
\hline
\end{tabular}

Overall, ten CD structures were generated to study how different numbers of HP influence the structural behavior using REMD simulation. A detailed information of the REMD method is given elsewhere [23,24]. The REMD simulations were performed by Amber 14 package [30]. The parameters of $\beta C D$ and $H P \beta C D$ s were taken from the Glycam06 carbohydrate force field [31,32] with the solvation model based on generalized Born (GB) implicit solvent model, Igb5, which gives a suitable description of cyclodextrin $\varepsilon C D$ as reported by Khuntawee et al. [33] and the smaller sizes of CDs $(\alpha C D-\delta C D$, unpublished data) relative to the available crystal structures and MD studies in explicit solvent model. The initial structures of $\beta C D$ and all HP $\beta C D$ s were fully minimized with 2000 steps of the steepest descent method, followed by 1000 steps of conjugated gradient method to relax the structures before simulation. The REMD simulations were performed for $30 \mathrm{~ns}$ per replica including equilibration step for $5 \mathrm{~ns}$, and the conformations at all temperatures were collected every 1 ps for 25 ns. The temperature distribution and the number of replicas were tested to obtain reasonable replica exchange simulation. The overlapping between the potential energy distributions of eight replicas is shown in Figure S1 of the supplementary materials. The results confirm that the temperature from $269.5 \mathrm{~K}$ to $570.9 \mathrm{~K}$ with interval steps around $30-60 \mathrm{~K}$ is proper for the present case.

The structural distortion of all HP $\beta C D$ s were analyzed by distances analysis as defined in Figure 3 . Firstly, the distances of adjacent glucopyranose units, $d_{1(i)}\left[\mathrm{O} 2_{(\mathrm{i})}-\mathrm{O} 3_{(\mathrm{i}+1)}\right]$ is defined as the distance between the secondary hydroxyl groups related to intramolecular hydrogen bonds of the wider CD rim, as labeled in Figure 3a. Secondly, the distance between the glycosidic oxygen atoms, $d_{2(i)}\left[\mathrm{O} 4_{(i)}-\right.$ $\mathrm{O} 44_{(i+1)}$ ] is calculated for monitoring the ellipticity of the CDs. The probability distributions of the $d_{1(i)}$ and $d_{2(i)}$ were calculated using equation 1 in terms of free energy.

$$
F(x, y)=-k_{B} T \log [P(x, y)]
$$

where $k_{B}$ is the Boltzmann constant, $T$ is the absolute temperature, and $P(x, y)$ is the probability of $x$ for $d_{1(i)}\left[\mathrm{O} 2_{(\mathrm{i})}-\mathrm{O} 33_{(i+1)}\right]$ and $y$ for $d_{2(i)}\left[\mathrm{O} 4_{(\mathrm{i})}-\mathrm{O} 4_{(i+1)}\right]$ distances.

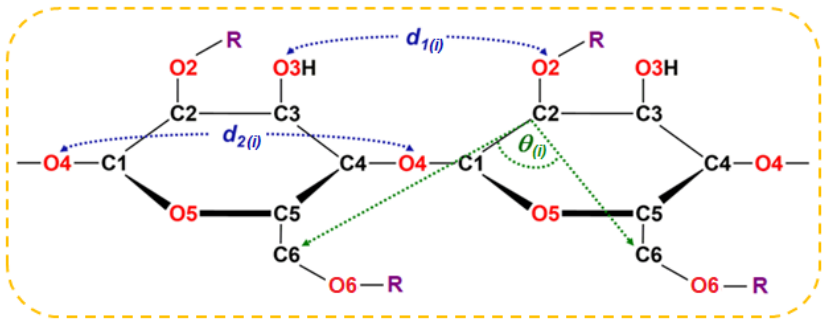

(a)

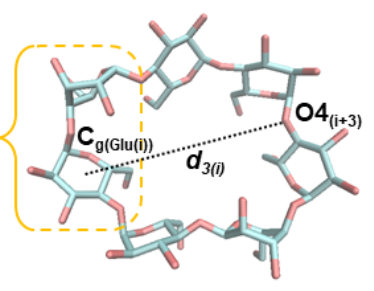

(b)

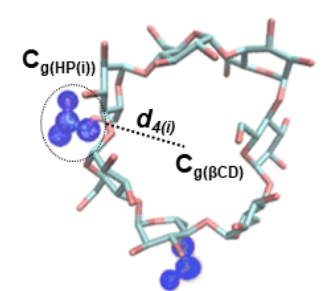

(c)

Figure 3. (a) $\mathrm{CD}$ fragment showing the atomic labels and important structural parameters, $d_{1(i)}\left[\mathrm{O} 2_{(\mathrm{i})}-\right.$ $\left.\mathrm{O} 3_{(i+1)}\right], d_{2(i)}\left[\mathrm{O} 4_{(i)-}-\mathrm{O} 4_{(i+1)}\right]$ and $\theta_{i)}\left[\mathrm{C} 6_{(i)}-\mathrm{C} 2_{(i+1)}-\mathrm{C}_{(i+1)}\right]$. (b) Set of diameters, $d_{3(i)}\left[\mathrm{C}_{\mathrm{g}(\mathrm{Glu}(\mathrm{i}))}-\mathrm{O} 44_{(i+3)}\right]$ for

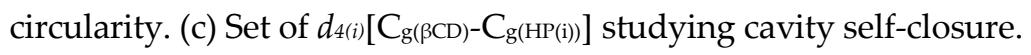

Next, the flips of glucose subunits for various conformations were monitored by angle between a pair of adjacent glucose units, $\theta_{i}\left[\mathrm{C} 6_{(i)}-\mathrm{C} 2_{(i+1)}-\mathrm{C} 6_{(i+1)}\right]$, as defined in Figure 3a. Without the flipping, the $\mathrm{C} 6$ atoms are in the same site with the adjacent unit. Thus, the $\theta_{i j}\left[\mathrm{C} 6_{(i)}-\mathrm{C}_{(i+1)-}-\mathrm{C}_{(i+1)}\right]$ values in no-flip 
structures are less than 90 degree. In contrast, when the unit is flipped, the C6 atoms were opposite to those of adjacent unit and its $\theta_{(i)}\left[\mathrm{C} 6_{(i)}-\mathrm{C}_{(i+1)}-\mathrm{C} 6_{(i+1)}\right]$ is higher than 90 degree. In addition, radius of gyration $\left(R_{g}\right)$ were calculated. The $R_{g}$ represents the mass weighted scalar length of each atom from the center of gravity of the molecule calculated using equation 2 .

$$
R_{g}=\sqrt{\frac{\sum_{i=1}^{N} m_{i}\left(r_{i}-r_{c m}\right)^{2}}{\sum_{i=1}^{N} m_{i}}}
$$

where $N$ is the number of atoms, $m_{i}$ is the mass of atom $i, r_{i}$ is the Cartesian position vector of atom $i$ and $r_{c m}$ is the center of mass of CD.

The next parameter is a modified circularity $(C)$, which is a dimensionless shape factor based on our previous work [34]. Here, we measured the diameter $d_{3(i)}\left[\mathrm{C}_{\mathrm{g}(\mathrm{Glu}(\mathrm{i}))}-\mathrm{O} 4_{(\mathrm{i}+3)}\right]$, which is a distance between center of mass of $i$-th glucose unit $\left(\mathrm{C}_{g(\mathrm{Glu}(\mathrm{i}))}\right)$ and the glycosidic oxygen atom of the opposite glucose unit $(\mathrm{O} 4(i+3))$ as depicted in Figure $3 b$. There are seven $d_{3(i)}$ parameters for each HP $\beta C D$ s snapshot since there are seven glucose subunits in $\beta C D$ s. Hence, $C$ is defined as:

$$
C=\frac{\min _{i \in\{1 \ldots 7\}}\left(d_{3(i)}\right)}{\max _{i \in\{1 \ldots 7\}}\left(d_{3(i)}\right)}
$$

Note that the value of $C$ is in a range of $0<C \leq 1$. If $C$ is equal to 1.0 where $\min \left(d_{3(i)}\right)=\max \left(d_{3(i)}\right)$, it means that the cavity has a perfect circular shape. However, when $C$ becomes deviated from 1.0, this is an indication for conformational changes to an elliptical shape. Finally, in order to study the cavity self-closure of HP $\beta C D$ s, we define the $d_{4(i)}\left[\mathrm{C}_{\mathrm{g}(\beta \mathrm{CD})}-\mathrm{C}_{\mathrm{g}(\mathrm{HP}(\mathrm{i}))}\right]$ parameter which is the distance of center of mass between $\beta C D$ ring and each $\mathrm{HP}$ group as demonstrated in Figure 3c. All parameters were analyzed from 25,000 snapshots taken from REMD simulations. The analyzed results at $300 \mathrm{~K}$ will be presented and discussed in the next section while those of other temperatures are provided in the supplementary materials.

\section{Results and Discussion}

\subsection{Structural Analysis}

\subsubsection{Structural Distortion of Glucose Units in the HP $\beta C D$ s}

To investigate the conformational changes of $\beta C D$ and all selected HP $\beta C D$, the probability distributions of $d_{1(i)}\left[\mathrm{O} 2_{(\mathrm{i})}-\mathrm{O} 3_{(\mathrm{i}+1)}\right]$ and $d_{2(i)}\left[\mathrm{O} 4_{(\mathrm{i})}-\mathrm{O} 4_{(\mathrm{i}+1)}\right]$ distances for all models at $300 \mathrm{~K}$ were calculated in terms of free energy using equation 1 and are plotted in Figure 4a. The contour graphs give the values of the distance probability where the denoted darkest blue color (ranked from dark red to blue) represents the lowest free energy.

In the case of $\beta C D$, three conformational minima (M1, M2 and M3) were detected as shown in Figure $4 \mathrm{a}(1)$, which is in agreement with previous results from MD in aqueous solution [29]. The most likely distribution at M1 was found with the $d_{1(i)}$ and $d_{2(i)}$ distances around 3.5 and $4.5 \AA$, respectively, describing the almost perfect conical shape of the $\beta C D$ ring. The second and third main population were $\mathrm{M} 2$ and $\mathrm{M} 3$ with $d_{1(i)} / d_{2(i)}$ around $5.5 \AA / 4.5 \AA$ and $5.0 \AA / 5.5 \AA$, respectively. When comparing $\beta C D$ with a large-ring CD ( $\varepsilon C D)$ by the REMD method [33], three important minima were found to be similar with $\beta C D$ results but the $M 3$ population of $\varepsilon C D$ was higher than that of $\beta C D$ due to the big size and high flexibility of the macroscopic ring, having a higher probability to flip the glucopyranose units in the large-ring system. 


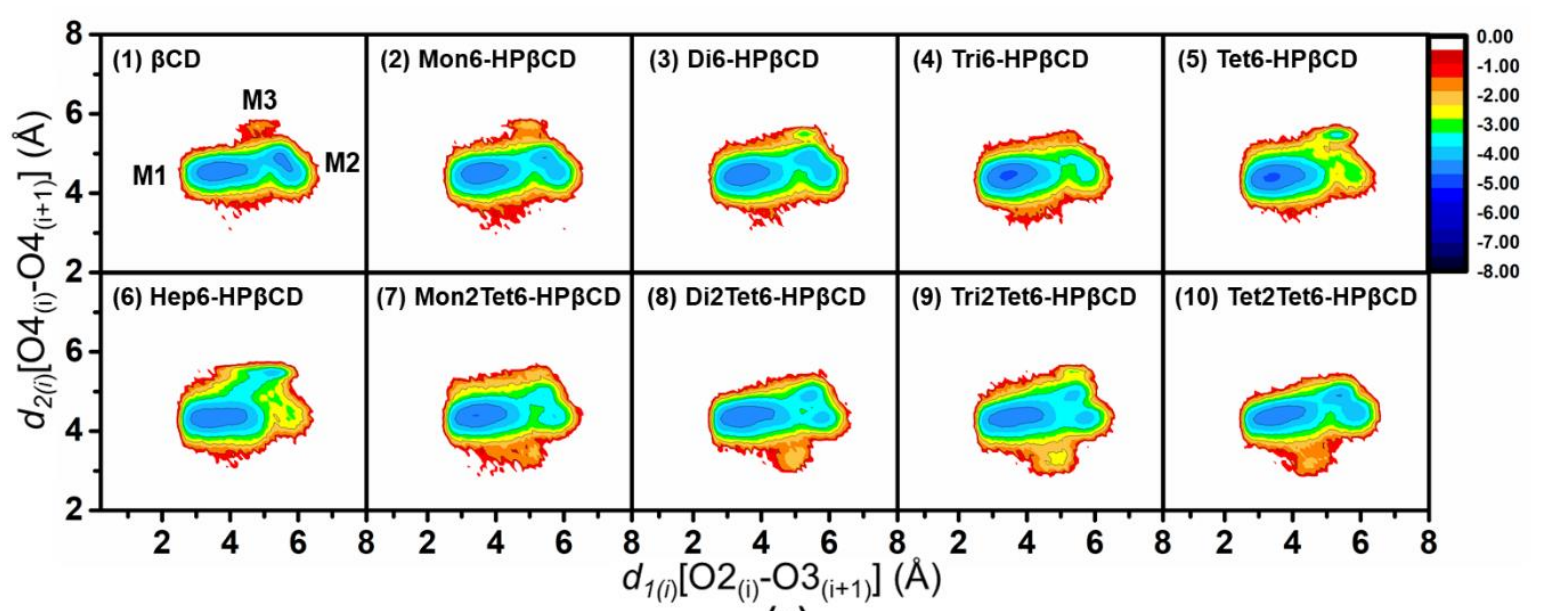

(a)

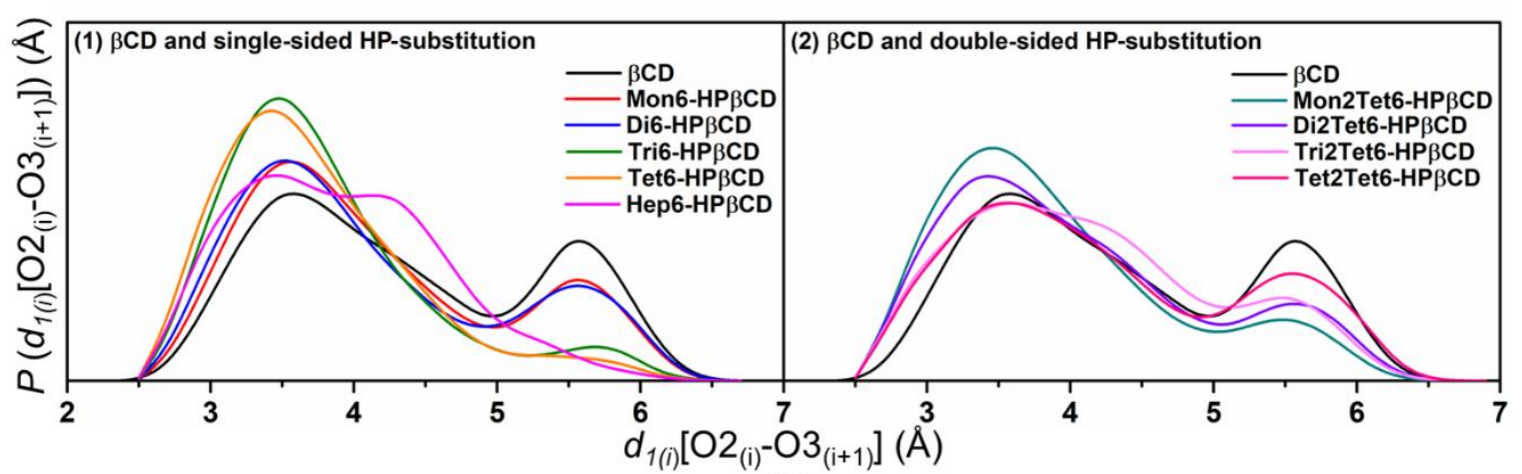

(b)

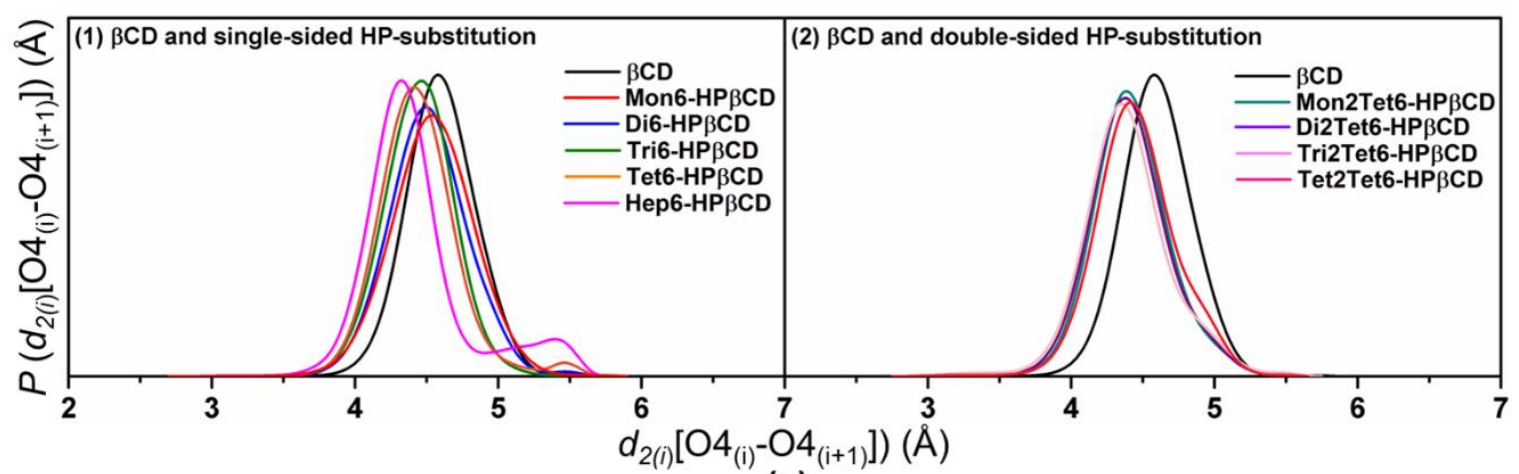

(c)

Figure 4. (a) Contour graphs of the probability distributions in terms of free energy, (b) the probability of the $d_{1(i)}$, and (c) the probability of the $d_{2(i)}$ from overall 25,000 snapshots at $300 \mathrm{~K}$ of

REMD simulations with the glycam 06 force field for $\beta C D$ and HP $\beta C D$ s.

From all free energy plots of HP $\beta C D$ s with single- and double-sided HP-substitutions, the $d_{2(i)}\left[\mathrm{O} 4_{(\mathrm{i})}-\mathrm{O} 4_{(\mathrm{i}+1)}\right]$ values fluctuated in the range of 4.0-5.0 $\AA$ implying that the backbone structures of the $\mathrm{HP} \beta \mathrm{CD}$ were not much affected by the HP-substitution groups. In contrast, it is noticeable that $d_{1(i)}\left[\mathrm{O} 2_{(\mathrm{i})}-\mathrm{O} 3_{(\mathrm{i}+1)}\right]$ is in wide range around 2.5-6.5 $\AA$. Therefore, we plotted the probability of the $d_{1(i)}\left[\mathrm{O} 2_{(i)}-\mathrm{O} 3_{(i+1)}\right]$ and the $d_{2(i)}\left[\mathrm{O} 4_{(i)-}-\mathrm{O} 4_{(i+1)}\right]$ as depicted in Figure $4 \mathrm{~b}$ and $4 \mathrm{c}$, respectively, in order to compare the conformational minima among the models. For HP $\beta C D$ s with single-sided HPsubstitutions, the most dominant population of $d_{1(i)}\left[\mathrm{O} 2_{(\mathrm{i})}-\mathrm{O} 33_{(i+1)}\right]$ for the Tri6- and Tet6-HP $\beta C D$ s was around $3.5 \AA$ indicating the narrow behavior of their secondary rim. Additionally, the conformational changes of other models were quite similar to the native $\beta C D$, except Hep6-HP $\beta C D$ with $d_{1(i)}\left[\mathrm{O} 2_{(i)}-\right.$ O3 $3_{(i+1)}$ ] around 4.0-5.0 $\AA$. Consequently, M3 was found in Hep6-HP $\beta C D$ higher than $\beta C D$ s and the other 6-HP $\beta C D$ s, resulting from steric hindrance of seven HP groups at the primary rim.

In the case of HP $\beta C D$ s with double-sided HP-substitution, the conformational changes were quite similar to the $\beta C D$, in which three main probability distributions were found following this 
order: $\mathrm{M} 1>\mathrm{M} 2>\mathrm{M} 3$. When increasing the number of $\mathrm{HP}$ at $\mathrm{O} 2$ positions, $\mathrm{M} 1$ population decreased while M2 population increased as depicted in Figure $4 \mathrm{a}(7)-4 \mathrm{a}(10)$ related with the distribution of $d_{1(i)}\left[\mathrm{O} 2_{(\mathrm{i})}-\mathrm{O} 3_{(\mathrm{i}+1)}\right]$ (Figure $4 \mathrm{~b}(2)$ ). In addition, the new probability distribution with $d_{1(i)} / d_{2(i)}$ around 4.3 $\AA / 3.5 \AA$ is observed when adding HP group on the secondary rim, and this probability distribution is enhanced when increasing the number of $\mathrm{HP}$ at $\mathrm{O} 2$ positions.

The results indicate that the substitution of HP groups on narrow and/or wider rims affected directly to the distortion in $\beta \mathrm{CD}$ ring. It is a worth noting that the $\mathrm{M} 1$ population was dramatically increased, whilst M2 was decreased and M3 was completely disappeared by a complexation with low-water soluble compounds [35-37].

\subsubsection{Flipping of the HP $\beta C D$ s}

It is noticeable that the flips of glucose subunits were related directly with the distortion of the $\mathrm{CD}$ structure. As mention earlier, a flip of glucose units was counted when $\theta_{i)}\left[\mathrm{C} 6_{(i)}-\mathrm{C} 2_{(i+1)}-\mathrm{C} 6_{(i+1)}\right]$ is higher than 90 degree. According to $\theta_{i i}\left[\mathrm{C} 6_{(i)}-\mathrm{C}_{(i+1)}-\mathrm{C}_{(i+1)}\right]$ analysis, the results can be divided into three main different flip-conformations: which are (1) no flip; (2) one flip; and (3) two flips. Example snapshot of flipped $\beta C D$ conformations are shown in Figure 5.
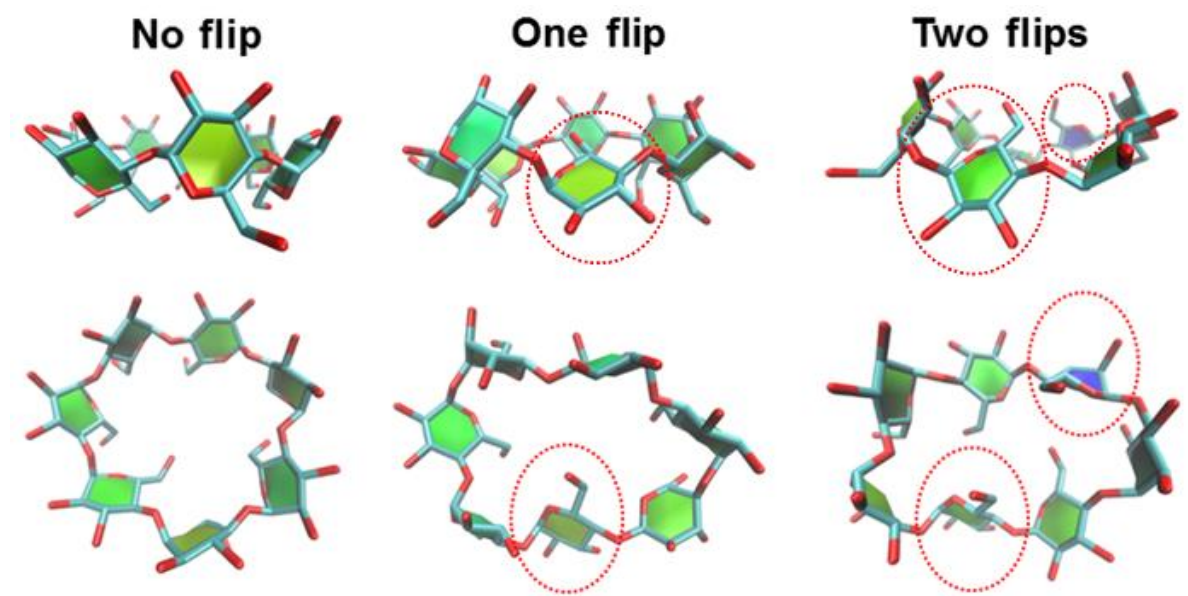

Figure 5. The flipped conformations of $\beta C D$ with different numbers of flip glucose subunits, which were selected from the simulation at $300 \mathrm{~K}$.

The probabilities of different numbers of flip glucose subunits for all models at $300 \mathrm{~K}$ are summarized in Table 2. The no-flip conformation is the main population in all models while other higher flips (one- and two-flip) are found to be of minor importance during the simulation. In all $\mathrm{HP} \beta C \mathrm{CD}$, more than $58 \%$ of no-flip conformations were observed whereas $\beta C D$ shows higher values of one- and two-flip conformations. The highest percentage of one- and two-flip conformations in $\beta C D$ corresponds to the long length of $d_{1(i)}\left[\mathrm{O} 2_{(\mathrm{i})}-\mathrm{O} 3_{(\mathrm{i}+1)}\right]$ detected around 5.0-6.0 $\AA$ in $\mathrm{M} 2$ population as shown in Figure $4 \mathrm{a}(1)$. With increasing number of HP only at O6 positions of glucose subunits, the no-flip population increased up to $75-78 \%$ in Tri6- and Tet6-HP $\beta C D$ s. Meanwhile, the one-flip population decreased from $35 \%$ to $21 \%$. These results were related with the large population of $d_{1(i)}\left[\mathrm{O} 2_{(\mathrm{i})}-\mathrm{O} 3_{(i+1)}\right]$ at $3.5 \AA$ for Tri6- and Tet6-HP $\beta C D$ s. For HP $\beta C D$ s with double-sided HP-substitution, the no-flip population also increased when compared with those of native $\beta C D$. However, the oneand two-flip populations of these of double-sided HP-substitution were higher than those of Tet6$\mathrm{HP} \beta C D$ s. In addition, the trends of these flips are not significantly different at other temperatures as shown in Table S1. The percentage of no flip angle correlates with M1, which is the most populated state during simulation, and the percentage of flip angle relates with M2 and M3, in which the glucopyranose subunits flip and the intramolecular hydrogen bonds of the wider CD rim disappear. Thus, glucose subunits in both 6-HP $\beta$ CDs and 2,6-HP $\beta$ CDs have lower chance to flip (22-31\% of flip angle) than in $\beta C D$ ( $42 \%$ of flip angle), which is in agreement with their inclusion efficiency reported in our previous study [22]. 
Table 2. The probability of different numbers of flip glucose subunits in $\beta C D$ and HP $\beta C D$ s using the flip angle parameter, $\theta_{i}\left[\mathrm{C} 6_{(i)}-\mathrm{C} 2_{(i+1)}-\mathrm{C} 6_{(i+1)}\right]$ at $300 \mathrm{~K}$ (criteria: higher value than 90 degree).

\begin{tabular}{lccc}
\hline \multirow{2}{*}{ Models } & \multicolumn{2}{c}{ The percentage of flip angle (\%) } \\
\cline { 2 - 4 } & No flip & One flip & Two flips \\
\hline$\beta C D$ & 58 & 35 & 7 \\
Single-sided HP-substitution & & \\
Mon6-HP $\beta C D$ & 69 & 28 & 3 \\
Di6-HP $\beta C D$ & 74 & 23 & 3 \\
Tri6-HP $\beta C D$ & 75 & 24 & 1 \\
Tet6-HP $\beta C D$ & 78 & 21 & 1 \\
Hep6-HP $\beta C D$ & 73 & 25 & 2 \\
Double-sided HP-substitution & & \\
Mon2Tet6-HP $\beta C D$ & 77 & 22 & 1 \\
Di2Tet6-HP $\beta C D$ & 75 & 24 & 1 \\
Tri2Tet6-HP $\beta C D$ & 70 & 28 & 2 \\
Tet2Tet6-HP $\beta C D$ & 74 & 24 & 2 \\
\hline
\end{tabular}

\subsubsection{Radius of gyration}

The flipping of the glucose subunits influences directly the distortion of the macrocyclic ring as shown in Figure 5 (top view of different flipped conformations). Therefore, the shape of all models was investigated in terms of radius of gyration $\left(R_{g}\right)$ and the results are shown in Figure 6 . The average of $R_{g}$ of native $\beta C D$, Mon6-, Di6-, Tri6-, Tet6- and Hep6-HP $\beta C D$ s are 6.18, 6.38, 6.39, 6.45, 6.55, and $6.85 \AA$, respectively, and those of Mon2Tet6-, Di2Tet6-, Tri2Tet6- and Tet2Tet6-HP $\beta C D s$ are 6.45, 6.46, 6.65 , and $6.85 \AA$. The results show that the average $R_{g}$ trends to increase when adding more HP groups which is similar to the $R_{g}$ results from a molecular dynamics simulation reported by Yong et al. [17]. However, for Hep6-HP $\beta C D$ and Tri2Tet6-HP $\beta C D$ with the same molar substitution (1.00), the average $R_{g}$ of Hep6-HP $\beta$ CD is higher than that of Tri2Tet6-HP $\beta C D$ because of the higher steric hindrance of the seven HP groups at the primary rim of Hep6-HP $\beta C D$. Overall, the $R_{g}$ in the substituted models was observed to be higher than the native $\beta C D$ because of fluctuation and steric hindrance of the HP groups.

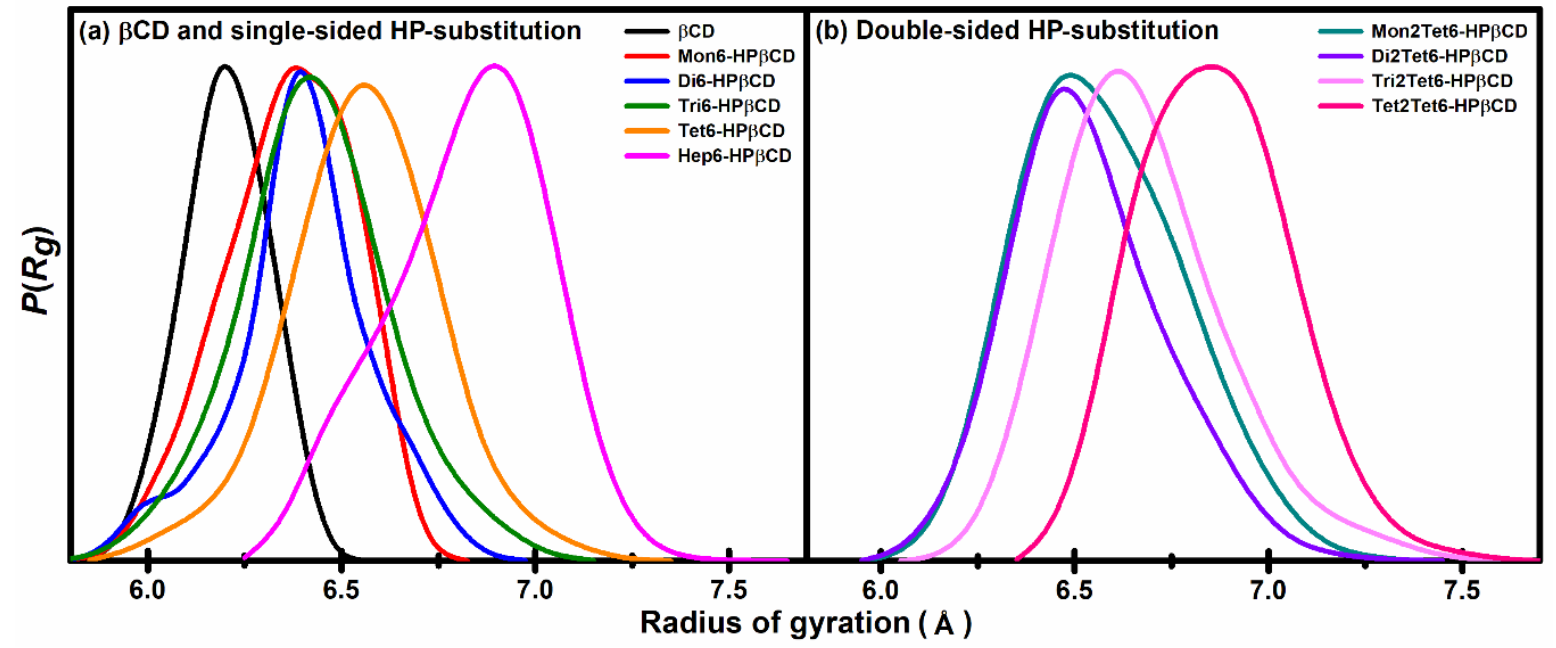

Figure 6. The probability of the radius of gyration for $\beta C D$ and all HP $\beta C D$ s at $300 \mathrm{~K}$. 


\subsubsection{Circularity}

To determine the effect of HP-substitutions on the geometry of CD cavity, we used the circularity $(C)$ calculated following equation 3 . The $C$ values of all models at $300 \mathrm{~K}$ are listed in Table $3 . \beta C D$ has the lowest $C(0.727 \pm 0.087)$ indicating that its conformational change is higher than those of other models. Moreover, the $C$ values of Tet2Tet6-HP $\beta C D$ s are very low too $(0.742 \pm 0.080)$. The reason for this more flexible conformation may be the result from the higher steric hindrance of HP groups on both $\mathrm{O} 2$ and $\mathrm{O} 6$ positions of the glucose subunits. In addition, taking the standard deviation into account, the $C$ values of Tri6- and Tet6-HP $\beta C D$ s are found to be $0.814 \pm 0.075$ and $0.815 \pm 0.076$, respectively. The data suggests that the cavity becomes more circular in shape when increasing the number of substituting HP at the O6 primary hydroxyl groups up to three or four residues corresponding to a high population of no-flip conformations. Although the population of no-flip conformations of Tri6-, Tet6- and Mon2Tet6-HP $\beta$ CDs as well as Di2Tet6-HP $\beta C D$ are quite similar (higher than 75\%), the $C$ values of Mon2Tet6- and Di2Tet6-HP $\beta C D$ s are lower than Tri6- and Tet6$\mathrm{HP} \beta \mathrm{CD}$ s due to the steric hindrance of HP groups leading to a distortion of some glucose subunits in no-flip conformation. For that reason, Tri6- and Tet6-HP $\beta C D$ s are highlighted as proper structures for forming inclusion complex with guest molecule indicated from their high circularity with lower possibility of flipping.

Table 3 The average and standard deviation of circularity $(C)$ of $\beta C D$ and $\mathrm{HP} \beta C D$ s at $300 \mathrm{~K}$ using REMD simulations.

\begin{tabular}{lc}
\hline \multicolumn{1}{c}{ Models } & $\boldsymbol{C}$ \\
\hline$\beta \mathrm{CD}$ & $0.727 \pm 0.087$ \\
Single-sided HP-substitution & \\
Mon6-HP $\beta C D$ & $0.746 \pm 0.085$ \\
Di6-HP $\beta C D$ & $0.751 \pm 0.086$ \\
Tri6-HP $\beta C D$ & $0.814 \pm 0.075$ \\
Tet6-HP $\beta C D$ & $0.815 \pm 0.076$ \\
Hep6-HP $\beta C D$ & $0.773 \pm 0.088$ \\
Double-sided HP-substitution & \\
Mon2Tet6-HP $\beta C D$ & $0.803 \pm 0.075$ \\
Di2Tet6-HP $\beta C D$ & $0.767 \pm 0.076$ \\
Tri2Tet6-HP $\beta C D$ & $0.785 \pm 0.074$ \\
Tet2Tet6-HP $\beta C D$ & $0.742 \pm 0.080$ \\
\hline
\end{tabular}

\subsection{Cavity self-closure}

The arrangement of HP groups during simulations influences the CD cavity accessibility directly. Some HP groups can point toward the CD interior leading to self-closure of CD cavity. The arrangement of the HP substituents at the glucose subunits were monitored via the distance of the

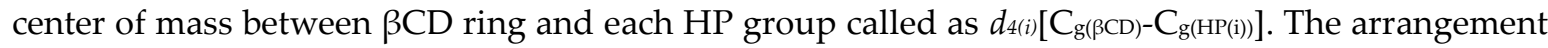
of HP for Mon6-HP $\beta C D$ is plotted in Figure 7 while those of other HP $\beta C D$ s are shown in Figure S2.

During REMD simulation of Mon6-HP $\beta C D$, almost $98 \%$ of HP groups point toward the CD

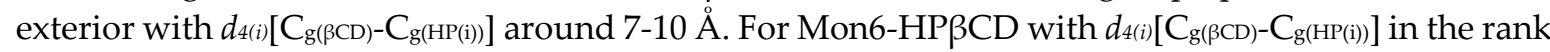
from 7 to $12 \AA$, the shape of Mon6-HP $\beta C D$ seems to be like a bowl (Figure $7 \mathrm{a})$. When the $d_{4(i)}\left[\mathrm{C}_{\mathrm{g}(\beta \mathrm{CD})}-\right.$ $\mathrm{C}_{\mathrm{g}(\mathrm{HP}(\mathrm{i}))]}$ is less than $3 \AA$, the HP group is hindered and rotated into the cavity of the $\beta C D$ ring leading to self-closure of $\mathrm{CD}$ cavity (Figure 7c). We also found that cavity self-closure is related with flipping

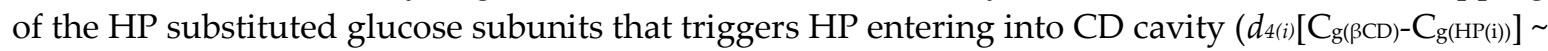
$1 \AA$ ). Some snapshots show that the substituents enter the cavity at the narrow rim with $d_{4(i)}\left[\mathrm{C}_{\mathrm{g}(\mathrm{BCD})-}\right.$ $\mathrm{C}_{\mathrm{g}(\mathrm{HP}(\mathrm{i}))]} \sim 2 \AA$. For that reason, we defined the self-closure of $\mathrm{CD}$ cavity or HP occupied in the $\mathrm{CD}$ cavity when $d_{4(i)}\left[\mathrm{C}_{\mathrm{g}(\beta \mathrm{CD})}-\mathrm{C}_{\mathrm{g}(\mathrm{HP}(\mathrm{i}))}\right]<3 \AA$. The numbers of $d_{4(i)}\left[\mathrm{C}_{\mathrm{g}(\beta \mathrm{CD})}-\mathrm{C}_{\mathrm{g}(\mathrm{HP}(\mathrm{i}))}\right]<3 \AA$ is called as $n(\mathrm{HP}$ inserted $)$ 
as illustrated in equation 4. For all REMD simulations of HP $\beta C D$ s at $300 \mathrm{~K}, n(\mathrm{HP}$ inserted $)$ are plotted in Figure 8 with the probability in term of percentage of 25,000 snapshots. The results at the various temperatures are summarized in Table S2.

$$
n\left(\mathrm{HP}_{\text {inserted }}\right)=n\left(d_{4(i)}\left[\mathrm{C}_{\mathrm{g}(\beta \mathrm{CD})}-\mathrm{C}_{\mathrm{g}(\mathrm{HP}(\mathrm{i}))}\right]<3 \AA\right)
$$

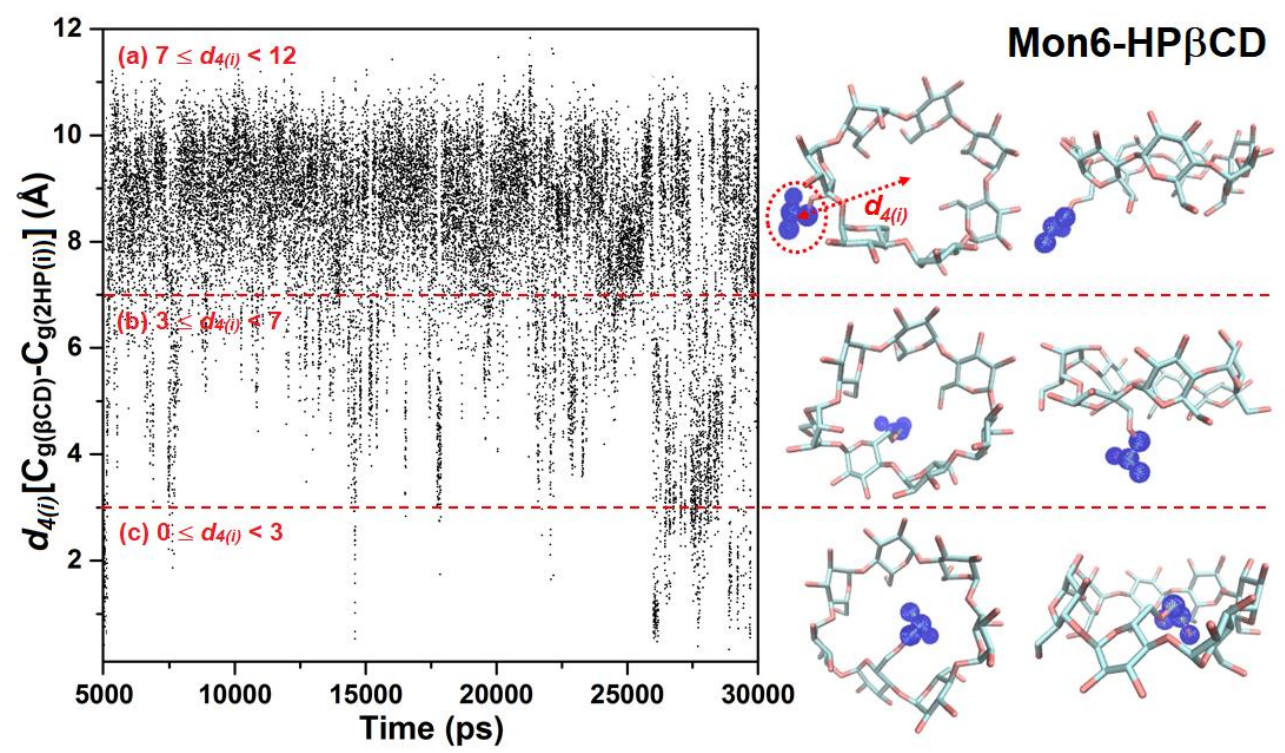

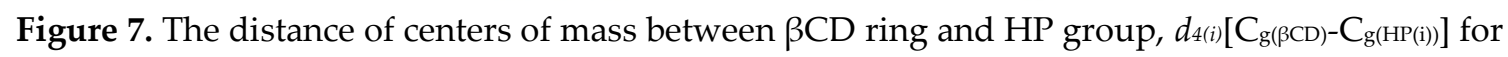
Mon6-HP $\beta C D$ at $300 \mathrm{~K}$.

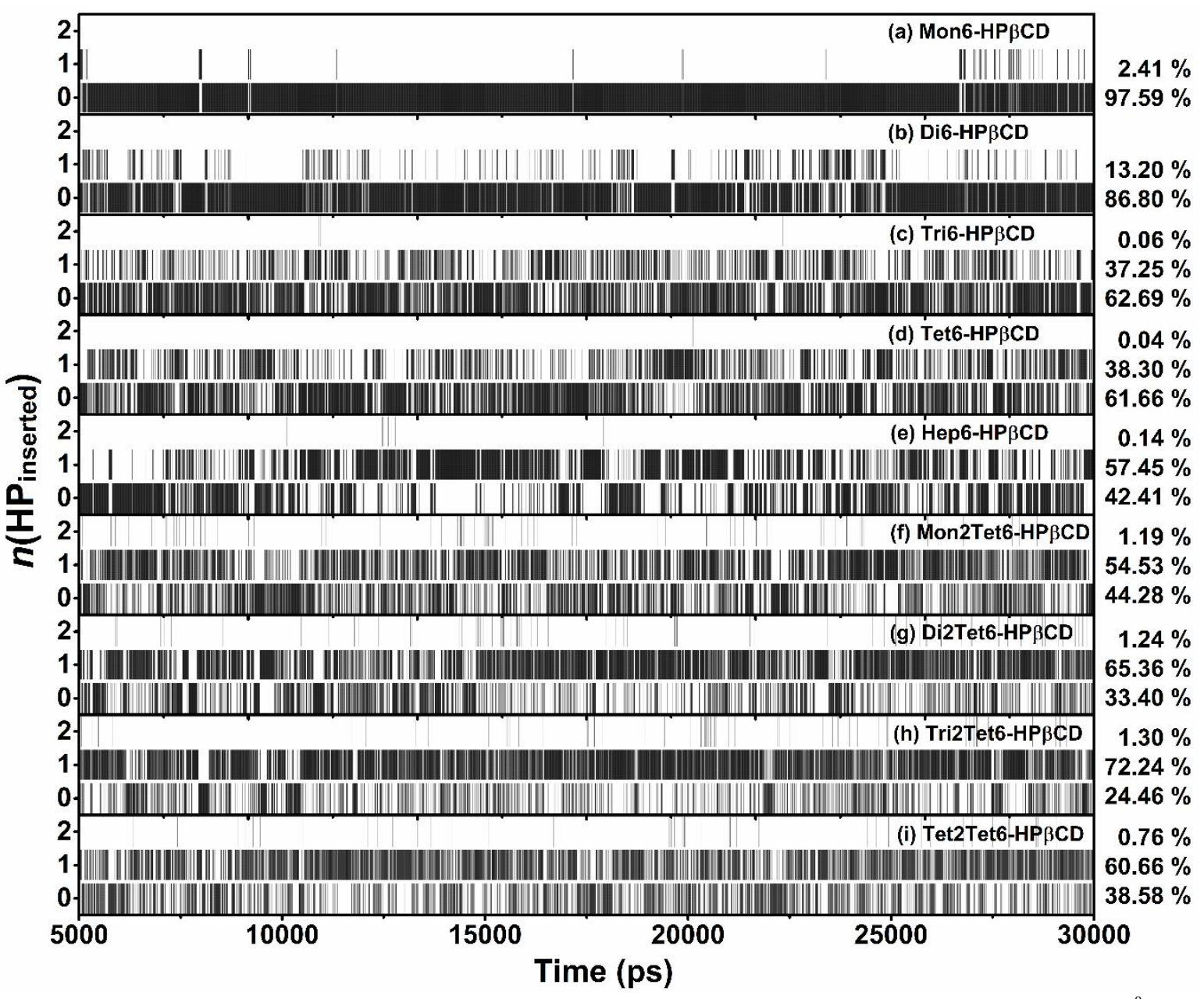

Figure 8. The probability of $n\left(\mathrm{HP}\right.$ inserted) from 25,000 snapshots (criteria: $d_{4(i)}\left[\mathrm{C}_{\mathrm{g}(\beta C \mathrm{CD})}-\mathrm{C}_{\mathrm{g}(\mathrm{HP}(\mathrm{i}))}\right]<3 \AA$ ) at $300 \mathrm{~K}$ for all HP $\beta C D$ s. 
For HP $\beta C D$ s with single-sided HP-substitution, it is noticeable that when the $n\left(\mathrm{HP}_{\text {inserted }}\right)>0$, the cavity self-closure occurs. Example snapshots of HP $\beta C D$ s with different numbers of $n(\mathrm{HP}$ inserted $)$ are given in Figure 9. As shown in the figure, the flipped HP substituted glucopyranose subunits and HP groups inserted to the hydrophobic cavity have led to the cavity self-closure HP $\beta C D$. When increasing number of HP groups, the percentage of cavity self-closure significantly increases from 2.41 to $57.59 \%$ in Hep6-HP $\beta C D$. It indicates that the cavity of the HP $\beta C D$ structure is almost blocked when the number of HP-substitution is increased. Furthermore, the maximum of $n(\mathrm{HP}$ inserted $)$ is equal to 2 even in the HP $\beta C D$ with high degree of HP-substitutions such as Hep6-HP $\beta C D$ and Tet2Tet6$\mathrm{HP} \beta \mathrm{CD}$.

(a) Single-sided HP substitution

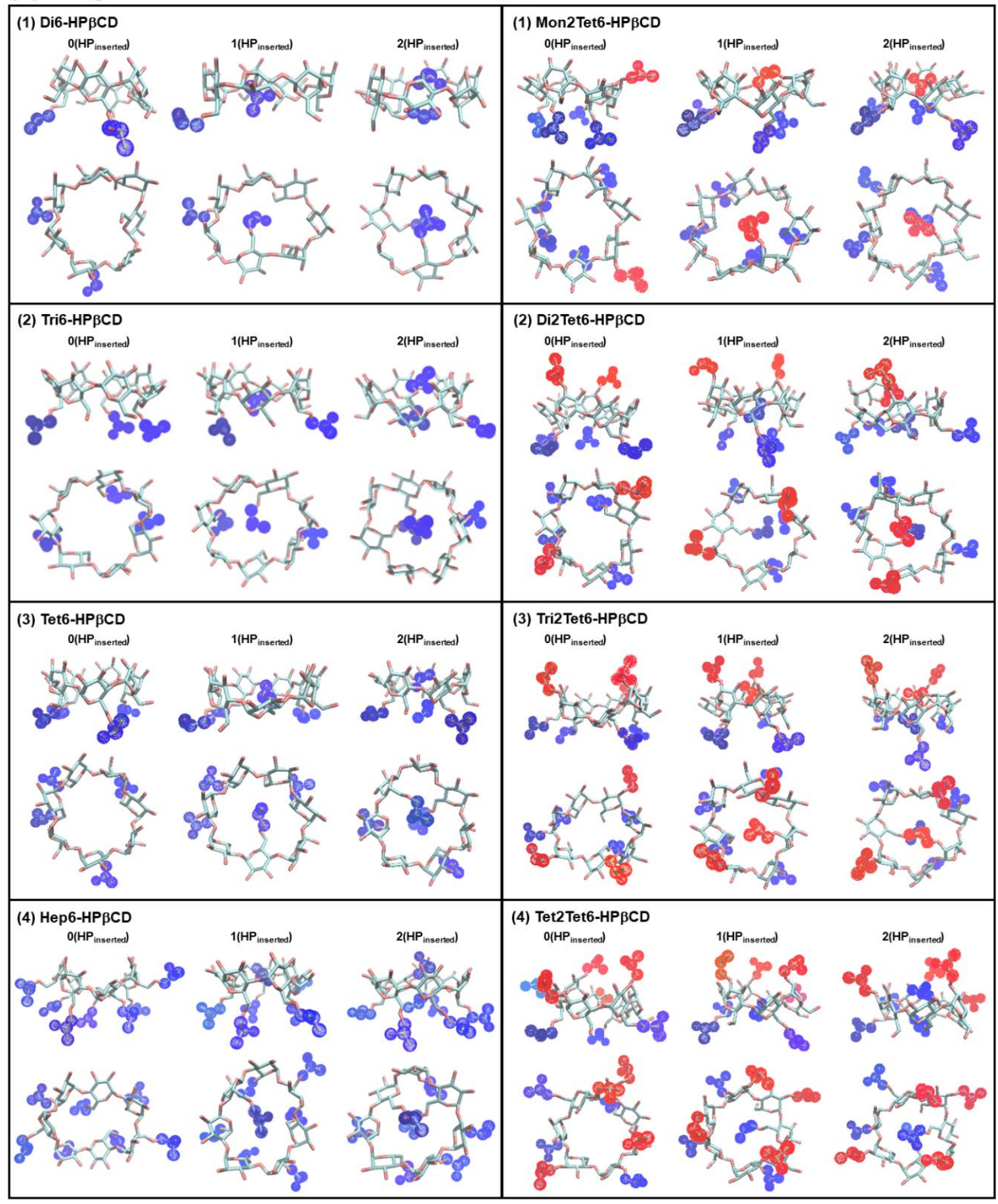

Figure 9. The example snapshots of different numbers of HP occupied in CD cavity (criteria:

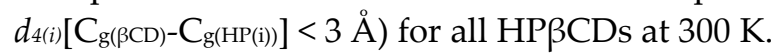


For HP $\beta C D$ s with double-sided HP-substitution, the HP groups both on narrow and wider rim of $\mathrm{HP} \beta C D$ can point toward into the cavity. For that reason, percentage of cavity self-closure is higher than those of single-sided HP-substitution because of the large fluctuation of their structures. When increasing the number of $\mathrm{HP}$ groups at $\mathrm{O} 2$ positions on Tet6-HP $\beta \mathrm{CD}$ from one to four, the probability of cavity self-closure rises dramatically from $38.34 \%$ (Tet6-HP $\beta C D$ ) to $55.72,66.60,73.54$ and $61.42 \%$ for Mon2Tet6-, Di2Tet6-, Tri2Tet6-, and Tet2Tet6-HP $\beta C D s$, respectively. With increasing temperatures, the glucose subunits distort, and HP groups fluctuate far from the center of mass of the $\beta C D$ ring leading to a lower probability of cavity self-closure at higher temperatures for all models (Table S2).

In summary, all data show that the HP-substitution strongly affects the cavity self-closure of $\mathrm{HP} \beta C D$, especially when the number of the HP substituents increases. To prevent the cavity selfclosure which might block the inclusion of the guest molecules, we suggest that the HP-substitution should be a single-sided substitution with the degree of substitution less than 0.57 such as Di-, Triand Tet6-HP $\beta C D$ s. However, it should be mentioned that the above results are only valid in cases, where there is no interaction between the cyclodextrins (at higher concentration). Moreover, the change of the dielectric properties e.g. by using solvent mixtures, might influence the conformations of the considered molecules as well as their conformational equilibria.

\section{Conclusions}

REMD simulations have been performed on HP $\beta C D$ s with various degrees of substitution (DS $=0.14-1.14$ ) to study the structural behavior and the effect of HP substituents. Several parameters that influence such changes have been identified. The circularity and the radius of gyration explain the size and shape cavity of the ring, and the flip angle and important distances describe the conformational change and the flexibility of the HP groups. The results show that HP $\beta C D$ s have a more pronounced conical shape than $\beta C D$, however the cavity self-closure occurs because some glucopyranoses with HP groups flip or distort followed by one or two HP groups coming close to CD cavity, thus hindering the drug inclusion. HP $\beta C D$ s with high DS are more likely to be blocked, while HP $\beta C D$ s with double-sided HP-substitution are even more probable to be blocked. Among the nine HP $\beta C D$ s, all analysis parameters point out that Tri- and Tet6-HP $\beta C D$ s with 3 and 4 HPsubstitutions on the primary rim have a distinctive conformation as mostly circular with low possibility of flipping and cavity enclosing. And thus these HP $\beta C D$ s could serve as more proper hosts for encapsulation of low-water soluble compounds.

Supplementary Materials: The following are available online at www.mdpi.com/xxx/s1, Figure S1: The overlapping between the potential energy distributions of each replica temperature ranging from $269.5 \mathrm{~K}$ to 570.9 K., Figure S2: The distance of centers of mass between $\beta C D$ ring and HP group, $d_{4(i)}\left[\mathrm{C}_{\mathrm{g}(\beta \mathrm{CD})}-\mathrm{Cg}_{(\mathrm{HP}(\mathrm{i}))]}\right.$ for (a) Di6HP $\beta C D$, (b) Tri6-HP $\beta C D$, (c) Tet6-HP $\beta C D$, (d) Hep6-HP $\beta C D$, (e) Mon2Tet6-HP $\beta C D$, (f) Di2Tet6-HP $\beta C D$, (g) Tri2Tet6-HP $\beta C D$, and (h) Tet2Tet6-HP $\beta C D$ at 300 K., Figure S3-S12: Contour graphs of the native $\beta C D$ (S3), Mon6-HP $\beta C D$ (S4), Di6-HP $\beta C D$ (S5), Tri6-HP $\beta C D$ (S6), Tet6-HP $\beta C D$ (S7), Hep6-HP $\beta C D$ (S8), Mon2Tet6$\mathrm{HP} \beta C D$ (S9), Di2Tet6-HP $\beta C D$ (S10), Tri2Tet6-HP $\beta C D$ (S11) and Tet2Tet6-HP $\beta C D$ (S12) probability distribution of 25,000 snapshots with the glycam06 force field at various temperatures., Figure S13: The radius of gyration in various temperatures for (a) $\beta C D$, (b) Mon6-HP $\beta C D$, (c) Di6-HP $\beta C D$, (d) Tri6-HP $\beta C D$, (e) Tet6-HP $\beta C D$, (f) Hep6-HPBCD, (g) Mon2Tet6-HPBCD, (h) Di2Tet6-HPBCD, (i) Tri2Tet6-HPBCD, and (j) Tet2Tet6-HPBCD., Table S1: The probability of different numbers of flip glucose subunits in $\beta C D$ and all $H P \beta C D$ s using flip angle parameter, $\theta_{i}\left[\mathrm{C}_{(i)}-\mathrm{C}_{(i+1)}-\mathrm{C}_{(i+1)}\right]$ in the various temperatures (criteria: more value than 90 degree) compared with classical MD simulation in the parenthesis., Table S2: The probability of overall snapshots with different numbers of HP occupied in CD cavity (criteria: $d_{4(i)}\left[\mathrm{C}_{\mathrm{g}(\beta \mathrm{CD})}-\mathrm{Cg}_{(\mathrm{HP}(\mathrm{i}))}\right]<3 \AA$ ) for all $\mathrm{HP} \beta \mathrm{CD}$ in the various temperatures.

Author Contributions: K.K. analyzed all data and wrote the original draft preparation. Some results of classical MD simulation were analyzed by J.K. T.R. and N.K. conceived the conceptualization. P.W., S.M., C.R., M.K., H.O., N.K. and T.R. supervised the project. All authors discussed the results and contributed to the final manuscript. 
Acknowledgments: This work was financially supported by the Thailand Research Fund (RSA5980069 to T.R. and RSA6180044 to N.K.), Chiang Mai University, and a Grant-in-Aid for Scientific Research on Innovative Areas in "Precisely Designed Catalysts with Customized Scaffolding" (JSPS KAKENHI Grant Number JP18H04233 to S.M.). K.K. thanks to W. Khuntawee, C. Hanpiboon, B. Nutho, and T. Saelee for resolving some technical problems. P.W. thanks Chulalongkorn University (CU) for a short-term visit grant. By travel grants for research visit, research reported in this publication was also supported by the ASEAN-European Academic University Network (ASEA-UNINET). The Center of Excellence Computational Chemistry (CU), and the Vienna Scientific Cluster (VSC-2) are gratefully thankful for computational facilities.

Conflicts of Interest: The authors declare no conflict of interest.

\section{References}

1 Uekama, K.; Hirayama, F.; Irie, T. Cyclodextrin Drug Carrier Systems. Chem. Rev. 1998, 98, $2045-2076$.

2 Hirayama, F.; Uekama, K. Cyclodextrin-based controlled drug release system. Adv. Drug Deliv. Rev. 1999, 36, 125-141.

3 Aachmann, F. L.; Otzen, D. E.; Larsen, K. L.; Wimmer, R. Structural background of cyclodextrin-protein interactions. Protein Eng. 2003, 16, 905-912.

4 Davis, M. E.; Brewster, M. E. Cyclodextrin-based pharmaceutics: past, present and future. Nat Rev Drug Discov 2004, 3, 1023-1035.

5 Challa, R.; Ahuja, A.; Ali, J.; Khar, R. K. Cyclodextrins in drug delivery: An updated review. AAPS PharmSciTech 2005, 6, E329-E357.

6 Loftsson, T.; Jarho, P.; Másson, M.; Järvinen, T. Cyclodextrins in drug delivery. Expert Opin Drug Deliv 2005, 2, 335-351.

7 Brewster, M. E.; Loftsson, T. Cyclodextrins as pharmaceutical solubilizers. Adv. Drug Deliv. Rev. 2007, 59, 645-666.

8 Marques, H. M. C. A review on cyclodextrin encapsulation of essential oils and volatiles. Flavour Frag. J. 2010, 25, 313-326.

9 Crini, G. Review: A History of Cyclodextrins. Chem. Rev. 2014, 114, 10940-10975.

10 Gould, S.; Scott, R. C. 2-Hydroxypropyl- $\beta$-cyclodextrin (HP- $\beta$-CD): A toxicology review. Food Chem. Toxicol. 2005, 43, 1451-1459.

11 Schönbeck, C.; Westh, P.; Madsen, J. C.; Larsen, K. L.; Städe, L. W.; Holm, R. Hydroxypropyl-Substituted $\beta$-Cyclodextrins: Influence of Degree of Substitution on the Thermodynamics of Complexation with Tauroconjugated and Glycoconjugated Bile Salts. Langmuir 2010, 26, 17949-17957.

12 Reyes-Reyes, M. L.; Roa-Morales, G.; Melgar-Fernández, R.; Reyes-Pérez, H.; Gómez-Oliván, L.; GonzalezRivas, N.; Bautista-Renedo, J.; Balderas-Hernández, P. Chiral recognition of abacavir enantiomers by (2hydroxy)propyl- $\beta$-cyclodextrin: UHPLC, NMR and DFT studies. J Incl Phenom Macrocycl Chem 2015, 82, 373-382.

13 Yuan, C.; Liu, B.; Liu, H. Characterization of hydroxypropyl- $\beta$-cyclodextrins with different substitution patterns via FTIR, GC-MS, and TG-DTA. Carbohydr. Polym. 2015, 118, 36-40.

14 Buvári-Barcza, Á.; Barcza, L. Influence of the guests, the type and degree of substitution on inclusion complex formation of substituted $\beta$-cyclodextrins. Talanta 1999, 49, 577-585.

15 Yuan, C.; Jin, Z.; Li, X. Evaluation of complex forming ability of hydroxypropyl- $\beta$-cyclodextrins. Food Chem. 2008, 106, 50-55.

16 Concha-Santos, S.; Pérez-Casas, S.; Brocos, P.; Piñeiro, Á. Testing the effect of the cavity size and the number of molecular substitutions in host-guest complexes formed by 2-hydroxypropyl-cyclodextrins and n-octyl$\beta$-d-glucopyranoside. J Chem Thermodyn 2013, 67, 112-119. 
Yong, C.; Washington, C.; Smith, W. Structural Behaviour of 2-Hydroxypropyl- $\beta$-Cyclodextrin in Water: Molecular Dynamics Simulation Studies. Pharm Res 2008, 25, 1092-1099.

Pitha, J.; Trinadha Rao, C.; Lindberg, B.; Seffers, P. Distribution of substituents in 2-hydroxypropyl ethers of cyclomaltoheptaose. Carbohydr Res 1990, 200, 429-435.

19 Trinadha Rao, C.; Pitha, J.; Lindberg, B.; Lindberg, J. Distribution of substituents in O-(2-hydroxypropyl) derivatives of cyclomalto-oligosaccharides (cyclodextrins): influence of increasing substitution, of the base used in the preparation, and of macrocyclic size. Carbohydr Res 1992, 223, 99-107.

20 Buvári-Barcza, Á.; Bodnár-Gyarmathy, D.; Barcza, L. Hydroxypropyl- $\beta$-cyclodextrins: Correlation between the stability of their inclusion complexes with phenolphthalein and the degree of substitution. J. Inclusion Phenom. Mol. Recognit. Chem. 1994, 18, 301-306.

21 Malanga, M.; Szemán, J.; Fenyvesi, É.; Puskás, I.; Csabai, K.; Gyémánt, G.; Fenyvesi, F.; Szente, L. “Back to the Future": A New Look at Hydroxypropyl Beta-Cyclodextrins. J. Pharm. Sci. 2016, 105, 2921-2931.

22 Nutho, B.; Nunthaboot, N.; Wolschann, P.; Kungwan, N.; Rungrotmongkol, T. Metadynamics supports molecular dynamics simulation-based binding affinities of eucalyptol and beta-cyclodextrin inclusion complexes. RSC Adv. 2017, 7, 50899-50911.

23 Sugita, Y.; Okamoto, Y. Replica-exchange molecular dynamics method for protein folding. Chem Phys Lett 1999, 314, 141-151.

24 Hukushima, K.; Nemoto, K. Exchange Monte Carlo Method and Application to Spin Glass Simulations. J. Phys. Soc. Jpn. 1996, 65, 1604-1608.

25 Hansmann, U. H. E. Parallel tempering algorithm for conformational studies of biological molecules. Chem Phys Lett 1997, 281, 140-150.

26 Nymeyer, H.; Gnanakaran, S.; García, A. E.: Atomic Simulations of Protein Folding, Using the Replica Exchange Algorithm. In Methods in Enzymology; Academic Press, 2004; Vol. Volume 383; pp 119-149.

27 Cheng, X.; Cui, G.; Hornak, V.; Simmerling, C. Modified Replica Exchange Simulation Methods for Local Structure Refinement. J. Phys. Chem. B 2005, 109, 8220-8230.

28 Snor, W.; Liedl, E.; Weiss-Greiler, P.; Karpfen, A.; Viernstein, H.; Wolschann, P. On the structure of anhydrous $\beta$-cyclodextrin. Chem Phys Lett 2007, 441, 159-162.

29 Kicuntod, J.; Khuntawee, W.; Wolschann, P.; Pongsawasdi, P.; Chavasiri, W.; Kungwan, N.; Rungrotmongkol, T. Inclusion complexation of pinostrobin with various cyclodextrin derivatives. $J \mathrm{Mol}$ Graph Model 2016, 63, 91-98.

30 Case, D. A.; Babin, V.; Berryman, J. T.; Betz, R. M.; Cai, Q.; Cerutti, D. S.; Cheatham, T. E.; Darden, T. A.; Duke, R. E.; Gohlke, H.; Goetz, A. W.; Gusarov, S.; Homeyer, N.; Janowski, P.; Kaus, J.; Kolossváry, I.; Kovalenko, A.; Lee, T. S.; LeGrand, S.; Luchko, T.; Luo, R.; Madej, B.; Merz, K. M.; Paesani, F.; Roe, D. R.; Roitberg, A.; Sagui, C.; Salomon-Ferrer, R.; Seabra, G.; Simmerling, C. L.; Smith, W.; Swails, J.; Walker; Wang, J.; Wolf, R. M.; Wu, X.; Kollman, P. A.: Amber 14, 2014.

31 Kirschner, K. N.; Yongye, A. B.; Tschampel, S. M.; González-Outeiriño, J.; Daniels, C. R.; Foley, B. L.; Woods, R. J. GLYCAM06: A generalizable biomolecular force field. Carbohydrates. J. Comput. Chem. 2008, 29, 622655.

32 Tessier, M. B.; DeMarco, M. L.; Yongye, A. B.; Woods, R. J. Extension of the GLYCAM06 biomolecular force field to lipids, lipid bilayers and glycolipids. Mol Simul 2008, 34, 349-364.

33 Khuntawee, W.; Rungrotmongkol, T.; Wolschann, P.; Pongsawasdi, P.; Kungwan, N.; Okumura, H.; Hannongbua, S. Conformation study of $\varepsilon$-cyclodextrin: Replica exchange molecular dynamics simulations. Carbohydr. Polym. 2016, 141, 99-105. 
34 Khuntawee, W.; Kunaseth, M.; Rungnim, C.; Intagorn, S.; Wolschann, P.; Kungwan, N.; Rungrotmongkol, T.; Hannongbua, S. Comparison of Implicit and Explicit Solvation Models for Iota-Cyclodextrin Conformation Analysis from Replica Exchange Molecular Dynamics. J Chem Inf Model 2017, 57, 778-786.

35 Wongpituk, P.; Nutho, B.; Panman, W.; Kungwan, N.; Wolschann, P.; Rungrotmongkol, T.; Nunthaboot, N. Structural dynamics and binding free energy of neral-cyclodextrins inclusion complexes: molecular dynamics simulation. Mol Simul 2017, 43, 1356-1363.

36 Mahalapbutr, P.; Nutho, B.; Wolschann, P.; Chavasiri, W.; Kungwan, N.; Rungrotmongkol, T. Molecular insights into inclusion complexes of mansonone $\mathrm{E}$ and $\mathrm{H}$ enantiomers with various $\beta$-cyclodextrins. J Mol Graph Model 2018, 79, 72-80.

37 Mahalapbutr, P.; Thitinanthavet, K.; Kedkham, T.; Nguyen, H.; Theu, L. t. h.; Dokmaisrijan, S.; Huynh, L.; Kungwan, N.; Rungrotmongkol, T. A theoretical study on the molecular encapsulation of luteolin and pinocembrin with various derivatized beta-cyclodextrins. J. Mol. Struct. 2018 (in press). 